\title{
Cooperative study on the value of long term anticoagulation in patients with stroke and non-rheumatic atrial fibrillation
}

\author{
J LODDER, M S DENNIS, L VAN RAAK, L N JONES, C P WARLOW
}

\begin{abstract}
The benefits of long term anticoagulant treatment of patients with non-rheumatic atrial fibrillation and cerebral infarction were studied by comparing two series of patients with stroke from centres with different policies on anticoagulant treatment. The long term prognosis of $\mathbf{5 0}$ patients from the Oxfordshire community stroke project, who did not receive anticoagulants, was compared with that of $\mathbf{7 0}$ similar patients from Maastricht, who were treated with anticoagulants. After a mean follow up of 27 months there was no significant difference in either the rate of survival or the rate of recurrent stroke between the two groups.

These data suggest that any benefit of anticoagulation is modest. A large randomised trial is planned to establish whether long term anticoagulant treatment is of value and, if so, to what extent.
\end{abstract}

\section{Introduction}

Early treatment with anticoagulants in cardioembolic stroke is safe provided certain clinical and computed tomographic findings are taken into account..$^{1-5}$ The question whether either short or long term treatment with anticoagulants reduces mortality or the risk of

Department of Neurology, Academisch Ziekenhuis Maastricht, University of Limburg, PO Box 1918, 6201 BX Maastricht, The Netherlands

J LODDER, MD, neurologist

L VAN RAAK, BA

Department of Clinical Neurology, Radeliffe Infirmary, Oxford OX2 6HE M S DENNIS, MB, MRCP, senior registrar in general medicine and geriatrics

Department of Community Medicine and General Practice, University, Oxford OX2 6HE

L N JONES, BA, computer programmer

Department of Medical Neurology and Neurology Unit, Northern General Hospital, Edinburgh EH5 2DQ

C P WARLOW, MD, FRCP, professor of neurology

Correspondence to: Dr Lodder. recurrence of stroke remains unanswered, ${ }^{6.8}$ particularly in nonrheumatic atrial fibrillation, which is the most common potential source of cardiac emboli in patients with stroke, being present in about $15 \%$ of all patients with ischaemic strokes.?

Supporters of the use of anticoagulants point to the high risk of early recurrence of stroke and emphasise the positive results of nonrandomised short term studies. ${ }^{149}$ The only randomised study of anticoagulants yielded inconclusive results, perhaps because of the few patients studied and it was stopped early. ${ }^{2}$ Other studies failed to confirm the high risk of early recurrence of stroke and to show an excess risk of later recurrence in patients with non-rheumatic atrial fibrillation compared with those in sinus rhythm. ${ }^{1011}$ None of these studies, however, excluded the possibility that patients with stroke and non-rheumatic atrial fibrillation might benefit from long term treatment with anticoagulants. A randomised controlled trial of anticoagulants in secondary prevention of cardioembolic stroke might reduce this uncertainty but may not be feasible. ${ }^{12}$

Because of these uncertainties and the lack of reliable data on the long term follow up of patients with stroke and non-rheumatic atrial fibrillation receiving anticoagulants we followed up a series of 70 patients in Maastricht who had been entered into a prospective study of the risk of early anticoagulant treatment in cardioembolic stroke. ${ }^{3}$ These follow up data were compared with those from a series of 50 similar patients with stroke and non-rheumatic atrial fibrillation not receiving anticoagulants selected from the Oxfordshire community stroke project. ${ }^{612}$ These two populations were selected because of the different attitudes of clinicians towards anticoagulation; patients in Maastricht usually received anticoagulants whereas those in Oxford seldom received them.

\section{Patients and methods}

In March 1981 we started the prospective study by taking patients admitted consecutively to Maastricht University Hospital. The criteria for including and excluding patients and details of the anticoagulant regimen have been described elsewhere. ${ }^{313}$ Briefly, we included patients with a nonseptic embolic supratentorial infarct of the brain whose symptoms lasted longer than 24 hours. All patients were given early computed tomography before anticoagulant treatment was started to exclude haemorrhagic stroke and had electrocardiography to confirm atrial fibrillation. Cardiac embolism was assumed to be the cause of stroke in the presence of paroxysmal or 
chronic non-rheumatic atrial fibrillation; rheumatic heart disease; aortic or mitral valve stenosis or prosthesis; or myocardial infarction in the preceding two weeks. Anticoagulant treatment was monitored by measurements (Thrombotest) at intervals decided by the attending doctor; a value of $8-12 \%$ was aimed at. We advised that anticoagulants should be continued for as long as a potential source of cardioemboli existed, provided that no contraindications developed.

By October 1985, 100 patients had been entered into this study. We followed up all patients during April 1986 to obtain information about $(a)$ survival and date and cause of death if appropriate; $(b)$ date of any recurrent stroke; and $(c)$ duration of anticoagulant treatment and complications. The relevant information was obtained by contacting the patients' general practitioners and also from medical records held by hospitals, nursing homes, and rehabilitation centres. Details of the patients' anticoagulant treatment were taken from files kept by the regional thrombosis service. Only 70 of the original 100 patients were included in the study; the remainder were excluded because they had had a second stroke or because they had a cardioembolic source other than non-rheumatic atrial fibrillation.

The Oxfordshire community stroke project has been described elsewhere. ${ }^{14}$ Briefly, it is a four year prospective community based study of all patients with first ever strokes within a population of about 105000 . All patients were assessed prospectively by a neurologist participating in the study as soon after the stroke as possible, irrespective of whether the patient was admitted to hospital or remained at home. Diagnosis was based on the results of computed tomography or necropsy, or both, in about $90 \%$ of the cases. All patients were followed up regularly by research nurses with a standard questionnaire designed to detect, among other things, recurrent strokes. If a recurrence was suspected the patient was reassessed neurologically. Of the 675 patients with first ever strokes registered during the four years of the study, 104 were in atrial fibrillation (confirmed by electrocardiography) either before or at the time of the stroke.

From these 104 patients we selected patients with similar general characteristics to those in Maastricht but who had not received anticoagulants. Figure 1 summarises the selection procedure. We excluded patients in whom infarction had not been confirmed by computed tomography or necropsy, or both; those with primary intracerebral haemorrhage; and those with clinical or echocardiographic evidence of rheumatic heart

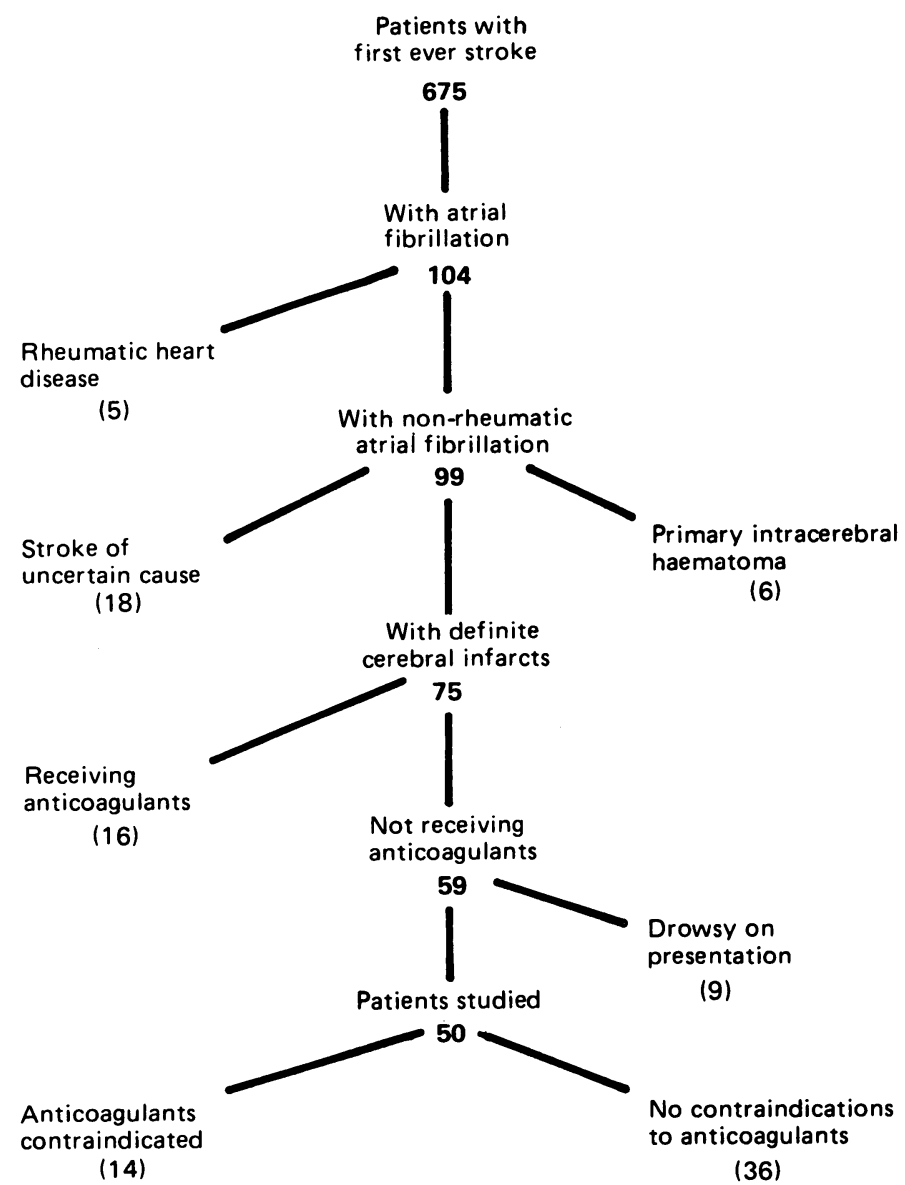

FIG 1-Selection of patients from Oxford community stroke project.
TABLE I-Age distribution of patients in Oxford and Maastricht. Values are numbers (percentages) of patients

\begin{tabular}{lrrrc}
\hline & \multicolumn{4}{c}{ Age (years) } \\
\cline { 2 - 5 } & $<70$ & $70-74$ & $75-79$ & $\geqslant 80$ \\
\hline $\begin{array}{l}\text { Oxford }(\mathrm{n}=50) \\
\text { Maastricht }(\mathrm{n}=70)\end{array}$ & $7(14)$ & $6(12)$ & $16(32)$ & $21(42)$ \\
\hline
\end{tabular}

TABLE II-Distribution of patients in Oxford and Maastricht by severity of stroke. Values are numbers (percentages) of patients

\begin{tabular}{lccr}
\hline & \multicolumn{3}{c}{ Severity of stroke } \\
\cline { 2 - 4 } & Mild & Moderate & Severe \\
\hline Oxford $(\mathrm{n}=50)$ & $23(46)$ & $18(36)$ & $9(18)$ \\
Maastricht $(\mathrm{n}=70)$ & $15(21)$ & $28(40)$ & $27(39)$ \\
\hline
\end{tabular}

disease. Of the remaining 59 patients, nine were drowsy on admission; in the Maastricht study such patients were not considered to be suitable for anticoagulant treatment because of their poor short term prognosis, and these nine patients were therefore also excluded. Fourteen of the 50 remaining patients had a contraindication to anticoagulant treatment such as dyspeptic symptoms, a history of peptic ulcer, or hypertension. After adjustment for age there was no significant difference in either risk of death $\left(\chi^{2}=0.51, p=0.47\right)$ or recurrent stroke $\left(\chi^{2}=0.03, p=0.87\right)$ between the patients with $(n=14)$ and without $(n=36)$ these contraindications. Even if the patients with contraindications did have a slightly worse prognosis (which cannot be excluded with such small numbers) including them in the study would tend to increase any apparent advantage of anticoagulant treatment. For these reasons we thought that it was reasonable to include these patients in the group that did not receive treatment.

The dates of first ever stroke, recurrent stroke, death, and last follow up together with information about age and severity of stroke were entered on to standard coding forms for both groups of patients. The data were entered into Oxford University's computer and analysed with a Fortran statistical program. Kaplan-Meier survival curves were constructed for various end points including death, recurrent stroke, and death and recurrent stroke. Outcome in the two groups was compared with the log rank test. ${ }^{15}$

The patients from the two centres differed both in their age distribution and also in the severity of their initial strokes. Patients in Maastricht had been categorised prospectively into three groups (mild, moderate, and severe) according to the method of Pessin et al. ${ }^{16}$ Patients in the Oxfordshire community stroke project were categorised retrospectively by the same criteria and with detailed assessments of both neurological impairment and disability. The groups were then retrospectively stratified by age and severity of stroke and estimates of the expected number of end points in each group calculated from the data adjusted for age and severity of stroke. The odds ratios were calculated to compare the prognosis in the two groups as described by Yusuf $e t a^{17}$; an odds ratio of less than one indicated a better prognosis in the Oxford group.

\section{Results}

The Maastricht series comprised 38 (54\%) men and 32 (46\%) women, a similar ratio to that in the Oxford group, in which there were 25 of each sex. Men and women were not significantly different in terms of risk of death $\left(\chi^{2}=0.04, p=0.82\right)$ or recurrent stroke $\left(\chi^{2}=0.05, p=0.82\right)$, although we could not exclude a slight difference because of the fairly small number of patients. Because of the lack of difference in outcome and the similar sex ratios in the two groups we did not adjust for sex in the subsequent analyses.

Table I shows the age distribution of the two groups; patients in the Oxford study were rather older than those in the Maastricht study. Table II shows the distribution of severity of stroke in the patients from the two centres; the proportion of mild strokes in Oxford was about double that in Maastricht. The mean length of follow up was 803 days (range 162-1467 days) in the Oxford group and 839 days (range 221-1877 days) in the Maastricht group. The Dutch patients received anticoagulant treatment for a mean of $99.5 \%$ of their follow up during which seven major and 13 minor haemorrhagic complications occurred. These complications were: intracerebral haemorrhage (non-fatal, one patient; fatal, two), fatal gastrointestinal haemorrhage (three), fatal pulmonary haemorrhage (one), haematuria (three), nosebleeds (four), and subcutaneous haematoma (six). 


\section{DEATHS AFTER FIRST STROKE}

During follow up 20 patients (40\%) in the Oxford group and $32(46 \%)$ in the Maastricht group died, six of the Dutch patients dying from complications of their treatment. Figure 2 shows the actuarial survival; the difference between the two groups was measured with the log rank and $\chi^{2}$ tests $\left(\chi^{2}=0.03, p=0.87\right)$.
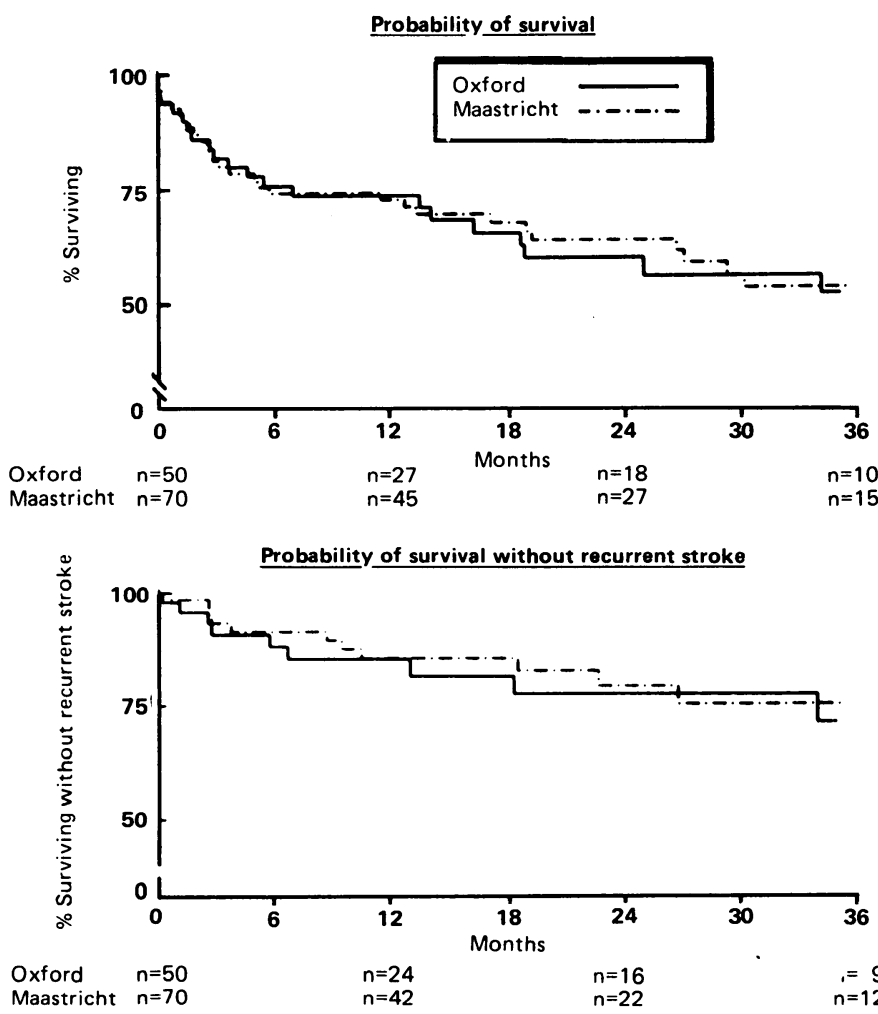

FIG 2-Kaplan-Meier survival curves of 50 patients not receiving anticoagulan treatment (Oxford community stroke project) and 70 patients receiving anticoagulants (Maastricht) after their first stroke. Numbers of patients at risk at different times are indicated.

Patients in each series were stratified into four age groups $(<70$, $70-74,75-79$, and $\geqslant 80$ ), and the data were analysed by a statistical test for trend to determine whether age was an explanatory prognostic variable. Interestingly, the expected survival was adversely affected by increasing age in the Oxford group (one tailed $p$ value for positive trend $=0.0003$ ) but not in the Maastricht group (one tailed $p=0 \cdot 39$ ). When both groups were analysed together there was a significant trend for increased mortality in older patients (one tailed $\mathrm{p}=0.008$ ).

Patients in both groups were stratified by severity of their initial stroke and the data analysed for trend; a similar pattern occurred, in which increasing severity of stroke was related to poor outcome in the Oxford group $(p=0.0014)$ but not in the Maastricht group $(p=0.34)$. When both groups were analysed together a significant trend was still present (one tailed $\mathrm{p}=0.02$ ). After stratification of patients by both age and severity of stroke the number of deaths in each group was compared with the number expected. The odds ratio-that is, the odds of dying for a patient not receiving anticoagulant treatment divided by the odds of dying for a patient receiving such treatment-was 1.0 (95\% confidence interval 0.50 to 1.98$)$, showing that survival in the two groups was not significantly different even when corrected for age and severity of stroke, although the upper $95 \%$ confidence limit was compatible with the hypothesis that anticoagulant treatment halved the risk of death and the lower limit that it doubled it.

\section{SURVIVAL WITHOUT RECURRENT STROKE}

During follow up nine patients (18\%) in the Oxford group and $14(20 \%)$ in the Maastricht group had a fatal or non-fatal stroke; three strokes in the group receiving treatment were due to primary intracerebral haemorrhage. Figure 2 shows the probability of remaining free of recurrent stroke, deaths from other causes having been subtracted from the denominator at the time of death. A log rank test showed no significant difference in outcome between the two groups $\left(\chi^{2}=0 \cdot 17, p=0.68\right)$. Stratifying patients from each series by age and severity of initial stroke, neither of which had a significant effect as a prognostic variable, did not make any difference; the odds ratio of $0.84(95 \%$ confidence interval 0.29 to 2.43 ) suggested a slight tendency for the patients who were not receiving anticoagulant treatment to have fewer recurrences, but the upper confidence limit was compatible with the hypothesis that anticoagulant treatment prevented over half of the recurrent strokes and the lower limit that it increased the risk of recurrent stroke threefold. A similar analysis was performed for the combination of end points stroke and death from all causes, recurrent strokes being counted only in patients who survived. It failed to show any significant difference between the two groups $\left(\chi^{2}=0.09, p=0.77\right)$, and this was not altered by adjustment for age and severity of stroke (odds ratio $=1 \cdot 0,95 \%$ confidence interval 0.53 to $1 \cdot 88$ ).

\section{Discussion}

The use of long term anticoagulant treatment in patients with stroke and non-rheumatic atrial fibrillation has often been discussed,,$^{1-1318}$ in part, possibly, because of the paucity of data on the effectiveness of such treatment. In two recent studies patients with stroke and non-rheumatic atrial fibrillation who were not given anticoagulant treatment were followed up. In one study $22 \%$ of the 68 patients had a recurrent stroke and $71 \%$ died mostly from other causes during five years of follow up ${ }^{11}$; in the other study 34 of 59 patients $(58 \%)$ died during a mean follow up of $3 \cdot 1$ years. ${ }^{18}$ Even allowing for the haemorrhagic complications that occurred in our patients receiving anticoagulants, they seem to have had a similar prognosis

In this non-randomised trial we selected patients from the Oxfordshire community stroke project who were as comparable as possible at presentation with the patients from Maastricht. Both series included only patients in whom a first ever ischaemic stroke and non-rheumatic atrial fibrillation had been confirmed; those with probable rheumatic heart disease were excluded. Age distribution and initial severity of stroke were, however, appreciably different in the two groups, which probably reflected different methods of selecting cases. All of the Dutch patients were admitted to hospital and selected as being suitable for anticoagulant treatment, which may have resulted in a more homogeneous group of patients than that obtained from the Oxfordshire community stroke project; this hypothesis is supported by the absence of a significant relation between age or severity of initial stroke and subsequent mortality in the Dutch but not the Oxford patients.

A small proportion (16/104) of patients with non-rheumatic atrial fibrillation in the Oxford group were excluded because they were already receiving anticoagulants. It might be argued that because those patients who would benefit most from treatment-that is, those at greatest risk of subsequent cardioembolic stroke-had already been effectively identified and excluded those remaining were likely to have a better than average prognosis. The decision to give anticoagulants seemed, however, to depend much more on the policies of doctors than on factors relating to the patient, and we doubt whether appreciable bias was introduced.

The patients receiving anticoagulants were followed up once only, by means of contact with general practitioners and review of case notes, whereas those in the Oxfordshire community stroke project were seen at regular intervals. This is unlikely to have influenced the survival data, but recurrences of minor stroke might have been missed in the treated patients. This bias, however, would tend to improve the prognosis for the group receiving treatment and would not have obscured an advantage of treatment.

Anticoagulant treatment is used primarily in cardioembolic stroke to reduce the risk of recurrent stroke, although it may affect long term survival by preventing both myocardial infarction and other thrombotic complications of stroke-for cxample, pulmonary emboli. Our results did not show any difference in risk of death or recurrence of stroke between the two groups even when disparities in age and severity of stroke were taken into account. Because of the sample size and the wide confidence intervals, however, the results are compatible with the hypothesis that long term anticoagulant treatment halves or doubles the risk of recurrent stroke and death. 
Thus any benefit of anticoagulants is likely to be modest, and a large randomised trial would be required to detect it reliably. Of course, any modest benefit would be offset by the complexity, side effects, and cost of the treatment; in any case, a rather small population of patients with stroke are eligible for such treatment. ${ }^{12}$ On the other hand, our negative results, based on a non-randomised comparison and with fairly wide confidence intervals, are unlikely to convince doctors who are already using long term anticoagulant treatment despite the lack of good data to support their opinion. To resolve this dilemma we are planning a three year randomised trial of 1200 patients to compare treatment with anticoagulants, aspirin, and placebo.

The Oxfordshire community stroke project is funded by the British Medical Research Council and the Chest, Heart, and Stroke Association. The Dutch Heart Foundation supported JL during a visit to Oxford for three months.

\section{References}

1 Furlan AS, Cavalier SJ, Hobbs RE, et al. Hemorrhage and anticoagulation after non-septic embolic brain infarction. Neurology 1982;32:280-2.

2 Cerebral Embolism Study Group. Immediate anticoagulation of embolic stroke: a randomized trial. Stroke 1983;14:668-76.
3 Lodder J, van der Lugt PJM. Evaluation of the risk of immediate anticoagulant treatment in patients with embolic stroke of cardiac origin. Stroke 1983;14:42-6.

4 Kelley RE, Berger JR, Alter M, et al. Cerebral ischemia and atrial fibrillation: prospective study. Neurology 1984;34:1285-91.

5 Martin GJ, Biller J. Non-septic cerebral emboli of cardiac origin. Arch Intern Med 1984;144: $1997-9$.

6 Starkey I, Warlow CP. The secondary prevention of stroke in patients with atrial fibrillation. Arch Neurol 1986;43:66-8.

7 Sherman OG, Hart RG, Easton JD. The secondary prevention of stroke in patients with atria fibrillation. Arch Neurol 1986;43:68-70.

8 Hachinski V. Atrial fibrillation and recurrent stroke. Arch Neurol 1986;43:70.

9 Koller RL. Recurrent embolic cerebral infarction and anticoagulation. Neurology 1982;32:283-5. 0 Norving B, Nilsson B. Cerebral embolism of cardiac origin; the limited possibilities of secondary prevention. Acta Neurol Scand 1986;73:520.

1 Gustafsson C, Britton M. Prognosis after brain infarction in patients with non-valvular atria fibrillation compared with sinus rhythm. Acta Neurol Scand 1986;73:520-1.

12 Sandercock P, Bamford J, Warlow C, Peto R, Starkey I. Is a controlled trial of long-term ora anticoagulants in patients with stroke and non-rheumatic atrial fibrillation worthwhile? Lance 1986;i:788-92.

13 Lodder J. A prospective study on the risk of immediate anticoagulation in cardiac embolic stroke. In: Stober T, Schimrigh K, Ganten D, Sherman D, eds. Central nervous system control of the heart. Boston: Martinus Nijhoff, 1986:245-8.

14 Oxfordshire Community Stroke Project. Incidence of stroke in Oxfordshire: first year's experience of a community stroke register. BrMed f 1983;287:713-7.

15 Peto R, Pike MC, Armitage $P$, et al. Design and analysis of randomized clinical trials requiring prolonged observation of each patient. II. Analysis and samples. Brf Cancer 1977;35:1-39.

16 Pessin MS, Hinton RC, Davis KR. Mechanisms of acute carotid stroke. Arch Neurol 1979;6: 245-52.

7 Yusuf S, Peto R, Lewis J, Collins R, Sleight P. Beta blockade during and after myocardia infarction: an overview of the randomized trials. Prog Cardiovasc Dis 1985;27:335-71.

18 Sage JI, Van Uitert RL. Risk of recurrent stroke in patients with atrial fibrillation and non-valvular heart disease. Stroke 1983:14:537-40.

(Accepted 16 December 1987)

\title{
Respiratory illness and home environment of ethnic groups
}

\author{
R J W MELIA, S CHINN, R J RONA
}

\begin{abstract}
Factors contributing to differences in the prevalences of respiratory symptoms and diseases among ethnic groups were studied in primary schoolchildren living in 20 inner city areas of England in 1983. The raised prevalences of respiratory symptoms in these groups were compared with results from a national representative sample of children studied in 1982. Data on age, sex, respiratory illness, and social and environmental variables were obtained by questionnaire for 4815 children living in inner cities. The children were classified as white, Afro-Caribbean, Urdu, Gujarati, Punjabi, other Asian, or "other." Significant differences in the prevalence of respiratory conditions were found among the ethnic groups after allowance was made for the effects of interfering variables. Except for asthma all conditions were most prevalent in Afro-Caribbeans and whites. In these two ethnic groups respiratory illness was significantly associated with belonging to a one parent family and the combined use of gas cookers and paraffin heaters at home.

Respiratory illness was found to vary in prevalence among ethnic groups but may be perceived differently by different groups. Further studies, measuring lung function, are necessary.
\end{abstract}

\footnotetext{
Department of Community Medicine, United Medical and Dental Schools of Guy's and St Thomas's Hospitals, London SE1 7EH

R J W MELIA, PHD, lecturer in epidemiology

$S$ CHINN, MA, senior lecturer in medical statistics

R J RONA, PHD, MFCM, senior lecturer in community medicine

Correspondence to: Dr Melia.
}

\section{Introduction}

The health of ethnic minority groups in the United Kingdom has been the subject of considerable discussion and concern during the past two decades. Inherited disorders such as sickle cell anaemia and illnesses such as rickets have been highlighted. ${ }^{12}$ Respiratory health has not been studied thoroughly, though a higher prevalence of respiratory illness has been reported in West Indians $s^{34}$ and respiratory illness in infants was reported to be more common among Bengalis than the indigenous population of an inner city area. ${ }^{5}$ The cause of these differences has not been studied in detail, although poor social circumstances are probably a factor in some ethnic groups, As respiratory disease in childhood has been linked with susceptibility to respiratory disease in later $\operatorname{life}^{6}$ it is important to investigate the causes of variation in respiratory health between children of different ethnic groups to identify preventive measures.

This study of primary schoolchildren investigated the prevalence of respiratory illness in ethnic groups in inner city areas and factors that contribute to differences in prevalence among the ethnic groups and between the groups from inner cities and a national sample of children.

\section{Subjects and methods}

In 1983 data on respiratory symptoms and diseases were collected in the national study of health and growth, a surveillance study of primary schoolchildren that included white, Afro-Caribbean, Indian, and Pakistani children from inner city areas, ${ }^{7}$ most of whom had spent most of their lives in Britain. Twenty wards in England with a high percentage of children of Afro-Caribbean and Indo-Pakistani ethnic origin and children living in inner city areas with a high level of overcrowding, unemployment, or lack of exclusive use of amenities had been selected, and about 350 children aged 5-11 from one or two schools close to the geographical centre of each ward had been studied. In 1983 the study investigated ethnic minorities for the first time. Several factors were studied in connection with respiratory disease but smoking was not one of them. To assess further the size of the 